Pacific

Journal of

Mathematics

EMBEDDINGS OF REDUCED FREE PRODUCTS OF OPERATOR ALGEBRAS

Etienne F. Blanchard and Kenneth J. Dykema 


\title{
EMBEDDINGS OF REDUCED FREE PRODUCTS OF OPERATOR ALGEBRAS
}

\author{
Etienne F. Blanchard and Kenneth J. Dykema
}

Given reduced amalgamated free products of $\mathrm{C}^{*}$-algebras $(A, \phi)=\underset{\iota \in I}{*}\left(A_{\iota}, \phi_{\iota}\right)$ and $(D, \psi)=\underset{\iota \in I}{*}\left(D_{\iota}, \psi_{\iota}\right)$, an embedding $A \hookrightarrow D$ is shown to exist assuming there are conditionalexpectation-preserving embeddings $A_{\iota} \hookrightarrow D_{\iota}$. This result is extended to show the existence of the reduced amalgamated free product of certain classes of unital completely positive maps. Finally, analogues of the above mentioned results are proved for amagamated free products of von Neumann algebras.

\section{Introduction.}

The reduced free product construction, and more generally the reduced amalgamated free product construction for $\mathrm{C}^{*}$-algebras, introduced independently by Voiculescu [21] and (somewhat less generally) Avitzour [1], has received much recent attention.

It is natural to ask: To what extent does the reduced free product construction satisfy a universal property, analogous to those for the free product of groups or the full free product of $\mathrm{C}^{*}$-algebras? Since the reduced free product of $\mathrm{C}^{*}$-algebras frequently gives rise to simple $\mathrm{C}^{*}$-algebras, (see $[\mathbf{1}],[\mathbf{1 5}]$, $[\mathbf{1 0}],[\mathbf{1 1}]$ and $[\mathbf{8}])$, it is clear that any universal property for the reduced free product should be quite a bit more restrictive in character than for the full free product; however, at first glance the following question still seems reasonable.

Question 1. If

$$
(A, \phi)=\underset{\iota \in I}{*}\left(A_{\iota}, \phi_{\iota}\right)
$$

is a reduced free product of $\mathrm{C}^{*}$-algebras, where the $\phi_{\iota}$ are states on the unital $\mathrm{C}^{*}$-algebras $A_{\iota}$ having faithful GNS representations, and if $D$ is a unital $\mathrm{C}^{*}$ algebra with a state $\psi$ and with unital $*$-homomorphisms $\kappa_{\iota}: A_{\iota} \rightarrow D$ such that

(i) $\psi \circ \kappa_{\iota}=\phi_{\iota}$ for every $\iota \in I$,

(ii) the family $\left(\kappa_{\iota}\left(A_{\iota}\right)\right)_{\iota \in I}$ is free with respect to $\psi$, 
does it follow that there is a $*$-homomorphism $\kappa: A \rightarrow D$ such that, denoting by $\alpha_{\iota}: A_{\iota} \rightarrow A$ the injective $*$-homomorphisms arising from the free product construction, $\kappa \circ \alpha_{\iota}=\kappa_{\iota}$ for every $\iota \in I$ ? (Note that $\kappa$ would necessarily be injective.)

Note that the homomorphism $\kappa$ exists if and only if the GNS representation $\pi_{\psi}: D \rightarrow \mathcal{L}\left(L^{2}(D, \psi)\right)$ of $\psi$ is faithful when restricted to the subalgebra of $D$ generated by $\bigcup_{\iota \in I} \kappa_{\iota}\left(A_{\iota}\right)$. As observed in $[\mathbf{1 6}, 1.3]$, the answer to Question 1 is "yes" if the state $\psi$ on $D$ is assumed to be faithful, (and a similar result holds in the amalgamated case). However, in general the answer is "no", as was shown by the elementary example $[\mathbf{1 6}, 1.4]$, (see also the erratum to $[\mathbf{1 6}])$.

The main result of this paper is an embedding result (Theorem 1.3) implying that the $*$-homomorphism $\pi$ in Question 1 does exist provided that the free subalgebras $\pi_{\iota}\left(A_{\iota}\right)$ lie in free subalgebras of $D$ that taken together generate $D$. Namely, we have the following property.

Property 2. Let $I$ be a set and for every $\iota \in I$ let $A_{\iota} \subseteq D_{\iota}$ be a unital inclusion of unital $\mathrm{C}^{*}$-algebras. Suppose $\phi_{\iota}$ is a state on $D_{\iota}$ such that the GNS representations of $\phi_{\iota}$ and of the restriction $\phi_{\iota} \uparrow_{A_{\iota}}$ are faithful. Consider the reduced free products of $\mathrm{C}^{*}$-algebras,

$$
\begin{aligned}
& (D, \phi)=\underset{\iota \in I}{*}\left(D_{\iota}, \phi_{\iota}\right) \\
& (A, \psi)=\underset{\iota \in I}{*}\left(A_{\iota}, \phi_{\iota} \uparrow_{A_{\iota}}\right) .
\end{aligned}
$$

Then there is a $*$-homomorphism $\pi: A \rightarrow D$ such that for every $\iota \in I$ the diagram

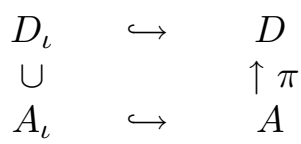

commutes, where the horizontal arrows are the inclusions arising from the free product construction.

This property was previously known under the additional assumption that every $\psi_{\iota}$ is faithful, which by [9] implies that $\psi$ is faithful on $D$; as noted after Question 1, this in turn implies the existence of $\pi$. Theorem 1.3 actually proves more generally a version of Property 2 for reduced amalgamated free products of $\mathrm{C}^{*}$-algebras. Such an embedding result is frequently useful for understanding reduced free product $\mathrm{C}^{*}$-algebras; it has been used in $[\mathbf{1 3}]$ and several times in [12].

We should point out that M. Choda has in [7] stated a theorem about reduced free products of completely positive maps which is more general than Property 2. However, her proof is incomplete, as it implicitly uses the full generality of Property 2 without justifying its validity. 
In $\S 1$, the main theorem about embeddings of reduced amalgamated free products of $\mathrm{C}^{*}$-algebras is proved. In $\S 2$, Choda's argument proving the existence of reduced free products of state-preserving completely positive maps is generalized to prove existence of reduced amalgamated free products of certain sorts of completely positive maps. In $\S 3$, we consider the reduced free product with amalgamation of von Neumann algebras and prove analogues of the results in $\S 1$ and $\S 2$ for von Neumann algebras.

Acknowledgements. Much of this work was done while K.D. was visiting the Institute of Mathematics of Luminy. He would like to thank the members of the Institute for their hospitality and for providing stimulating atmosphere during his visit. The authors would like to thank the referee for helpful comments.

\section{Embeddings.}

In this section we prove the main embedding result. We use the same notation as in [12] for the reduced amalgamated free product construction.

In the following lemma, with the reduced amalgamated free product of $\mathrm{C}^{*}$-algebras $(A, \phi)=\underset{\iota \in I}{*}\left(A_{\iota}, \phi_{\iota}\right)$ we view each $A_{\iota}$ as a $\mathrm{C}^{*}$-subalgebra of $A$ via the canonical embedding arising from the free product construction.

Lemma 1.1. Let $B$ be a unital $C^{*}$-algebra, let $I$ be a set and for every $\iota \in I$ let $A_{\iota}$ be a unital $C^{*}$-algebra containing a copy of $B$ as a unital $C^{*}$-subalgebra and having a conditional expectation $\phi_{\iota}: A_{\iota} \rightarrow B$ whose GNS representation is faithful. Let

$$
(A, \phi)=\underset{\iota \in I}{*}\left(A_{\iota}, \phi_{\iota}\right)
$$

be the reduced amalgamated free product. Then for every $\iota_{0} \in I$ there is a conditional expectation $\Phi_{\iota_{0}}: A \rightarrow A_{\iota_{0}}$ such that $\Phi_{\iota_{0}} \Upsilon_{A_{\iota}}=\phi_{\iota}$ for every $\iota \in I \backslash\left\{\iota_{0}\right\}$ and $\Phi_{\iota_{0}}\left(a_{1} a_{2} \cdots a_{n}\right)=0$ whenever $n \geq 2$ and $a_{j} \in A_{\iota_{j}} \cap \operatorname{ker} \phi_{\iota_{j}}$ with $\iota_{1} \neq \iota_{2}, \ldots, \iota_{n-1} \neq \iota_{n}$.

Proof. We let $E_{\iota}=L^{2}\left(A_{\iota}, \phi_{\iota}\right), \xi_{\iota}=\widehat{1_{A_{\iota}}} \in E_{\iota}, E_{\iota}=\xi_{\iota} B \oplus E_{\iota}^{\mathrm{o}}$. Then $A$ acts (by definition) on the Hilbert $B$-module

$$
E=\xi B \oplus \bigoplus_{\substack{n \geq 1 \\ \iota_{1}, \ldots, \iota_{n} \in I \\ \iota_{1} \neq \iota_{2}, \iota_{2} \neq \iota_{3}, \ldots, \iota_{n-1} \neq \iota_{n}}} E_{\iota_{1}}^{\mathrm{o}} \otimes_{B} E_{\iota_{2}}^{\mathrm{o}} \otimes_{B} \cdots \otimes_{B} E_{\iota_{n}}^{\mathrm{o}} .
$$

Identify the submodule $\xi B \oplus E_{\iota_{0}}^{\mathrm{o}}$ of $E$ with the Hilbert $B$-module $E_{\iota_{0}}$ and let $Q_{\iota_{0}}: E \rightarrow E_{\iota_{0}}$ be the projection. Then $\Phi_{\iota_{0}}(x)=Q_{\iota_{0}} x Q_{\iota_{0}}$ has the desired properties.

Explication 1.2. Consider the GNS representation $\left(\sigma, L^{2}\left(A, \Phi_{\iota_{0}}\right), \eta\right)=$ $\operatorname{GNS}\left(A, \Phi_{\iota_{0}}\right)$ associated with the conditional expectation $\Phi_{\iota_{0}}: A \rightarrow A_{\iota_{0}}$ 
found in Lemma 1.1. Since $A$ is the closed linear span of $B$ and the set of reduced words of the form $a_{1} a_{2} \cdots a_{n}$ where $a_{j} \in A_{\iota_{j}} \cap \operatorname{ker} \phi_{\iota_{j}}$ and $\iota_{j} \neq \iota_{j+1}$, we see that the Hilbert $A_{\iota_{0}}$-module in the GNS representation is

$$
L^{2}\left(A, \Phi_{\iota_{0}}\right)=A_{\iota_{0}} \oplus \bigoplus_{\substack{n \geq 1 \\ \iota_{1}, \ldots, \iota_{n} \in I \\ \iota_{1} \neq \iota_{2}, \ldots, \iota_{n-1} \neq \iota_{n} \\ \iota_{n} \neq \iota_{0}}} E_{\iota_{1}}^{\mathrm{o}} \otimes_{B} \cdots \otimes_{B} E_{\iota_{n}}^{\mathrm{o}} \otimes_{B} A_{\iota_{0}} .
$$

Moreover, the action $\sigma$ of $A$ on $L^{2}\left(A, \Phi_{\iota_{0}}\right)$ is determined by its restrictions $\sigma \uparrow_{A_{\iota}}$, which are easily described.

Let $\rho: A_{\iota_{0}} \rightarrow \mathcal{L}(\mathcal{V})$ be a unital $*$-homomorphism, for some Hilbert space V. Then $\sigma \otimes 1: A \rightarrow \mathcal{L}\left(L^{2}\left(A, \Phi_{\iota_{0}}\right) \otimes_{\rho} \mathcal{V}\right)$ is a $*$-homomorphism; it is the representation induced, in the sense of Rieffel [19], from $\rho$ up to $A$, with respect to the conditional expectation $\Phi_{\iota_{0}}$, and we will denote this induced representation by $\rho L^{A}$. We have the following explicit description of $\rho l^{A}$, obtained by tensoring (1) with $\otimes_{\rho} \mathcal{V}$ on the right. Writing $\mathcal{H}=$ $L^{2}\left(A, \Phi_{\iota_{0}}\right) \otimes_{\rho} \nu$ we have

$$
\mathcal{H}=\mathcal{V} \oplus \bigoplus_{\substack{n \geq 1 \\ \iota_{1}, \ldots, \iota_{n} \in I \\ \iota_{1} \neq \iota_{2}, \ldots, \iota_{n-1} \neq \iota_{n} \\ \iota_{n} \neq \iota_{0}}} E_{\iota_{1}}^{\mathrm{o}} \otimes_{B} \cdots \otimes_{B} E_{\iota_{n}}^{\mathrm{o}} \otimes_{\rho} \mathcal{V} .
$$

Moreover, the $*$-homomorphism $\sigma \otimes 1$ is determined by its restrictions

$$
\sigma_{\iota} \stackrel{\text { def }}{=}(\sigma \otimes 1) \uparrow_{A_{\iota}}: A_{\iota} \rightarrow \mathcal{L}(\mathcal{H}),
$$

given as follows. Consider the Hilbert spaces

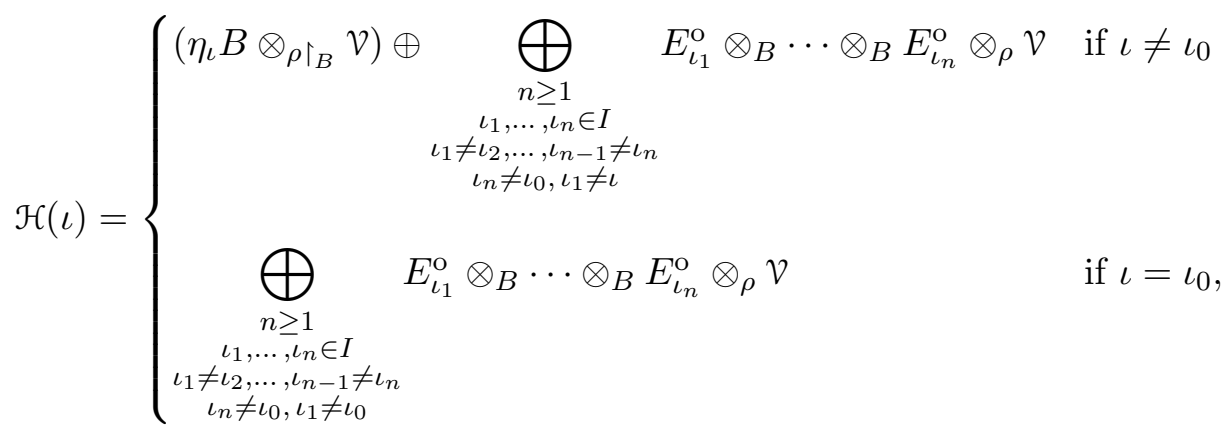

where $\eta_{\iota} B$ is just the Hilbert $B$-module $B$ with identity element denoted by $\eta_{\iota}$. If $\iota \in I \backslash\left\{\iota_{0}\right\}$ let

$$
W_{\iota}: E_{\iota} \otimes_{B} \mathcal{H}(\iota) \rightarrow \mathcal{H}
$$


be the unitary defined, using the symbol $\ddot{\otimes}$ to denote the tensor product in (3), by

$$
\begin{aligned}
& W_{\iota}: \xi_{\iota} \ddot{\otimes}\left(\eta_{\iota} \otimes v\right) \mapsto v \\
& \zeta \ddot{\otimes}\left(\eta_{\iota} \otimes v\right) \mapsto \zeta \otimes v \\
& \xi_{\iota} \ddot{\otimes}\left(\zeta_{1} \otimes \cdots \otimes \zeta_{n} \otimes v\right) \mapsto \zeta_{1} \otimes \cdots \otimes \zeta_{n} \otimes v \\
& \zeta \ddot{\otimes}\left(\zeta_{1} \otimes \cdots \otimes \zeta_{n} \otimes v\right) \mapsto \zeta \otimes \zeta_{1} \otimes \cdots \otimes \zeta_{n} \otimes v
\end{aligned}
$$

whenever $v \in \mathcal{V}, \zeta \in E_{\iota}^{\mathrm{o}}, \zeta_{j} \in E_{\iota_{j}}^{\mathrm{o}}$ and $\iota \neq \iota_{1}, \iota_{1} \neq \iota_{2}, \ldots, \iota_{n-1} \neq \iota_{n}, \iota_{n} \neq \iota_{0}$. Then for every $\iota \in I \backslash\left\{\iota_{0}\right\}$ and $a \in A_{\iota}$, we have

$$
\sigma_{\iota}(a)=W_{\iota}\left(a \otimes 1_{\mathcal{H}(\iota)}\right) W_{\iota}^{*} .
$$

Similarly, define the unitary

$$
W_{\iota_{0}}: \mathcal{V} \oplus\left(E_{\iota} \otimes_{B} \mathcal{H}\left(\iota_{0}\right)\right) \rightarrow \mathcal{H}
$$

by

$$
\begin{aligned}
W_{\iota_{0}}: & v \oplus 0 \mapsto v \\
& 0 \oplus\left(\xi_{\iota_{0}} \ddot{\otimes}\left(\zeta_{1} \otimes \cdots \otimes \zeta_{n} \otimes v\right)\right) \mapsto \zeta_{1} \otimes \cdots \otimes \zeta_{n} \otimes v \\
& 0 \oplus\left(\zeta \ddot{\otimes}\left(\zeta_{1} \otimes \cdots \otimes \zeta_{n} \otimes v\right)\right) \mapsto \zeta \otimes \zeta_{1} \otimes \cdots \otimes \zeta_{n} \otimes v
\end{aligned}
$$

Then

$$
\sigma_{\iota_{0}}(a)=W_{\iota_{0}}\left(\rho(a) \oplus\left(a \otimes 1_{\mathcal{H}\left(\iota_{0}\right)}\right)\right) W_{\iota_{0}}^{*} .
$$

Note that the above description is related to the construction of the conditionally free product, due to Bożejko and Speicher [5], (see also [4]).

Theorem 1.3. Let $B \subseteq \widetilde{B}$ be a (not necessarily unital) inclusion of unital $C^{*}$-algebras. Let $I$ be a set and for each $\iota \in I$ suppose

$$
\begin{aligned}
& 1_{\widetilde{A}_{\iota}} \in \widetilde{B} \subseteq \widetilde{A}_{\iota} \\
& \cup \\
& 1_{A_{\iota}} \in B \subseteq A_{\iota}
\end{aligned}
$$

are inclusions of $C^{*}$-algebras. Suppose that $\tilde{\phi}_{\iota}: \widetilde{A}_{\iota} \rightarrow \widetilde{B}$ is a conditional expectation such that $\tilde{\phi}_{\iota}\left(A_{\iota}\right) \subseteq B$ and assume that $\tilde{\phi}_{\iota}$ and the restriction $\tilde{\phi}_{\iota} \uparrow_{A_{\iota}}$ have faithful GNS representations, for all $\iota \in I$. Let

$$
\begin{aligned}
& (\tilde{A}, \tilde{\phi})=\underset{\iota \in I}{*}\left(\widetilde{A}_{\iota}, \tilde{\phi}_{\iota}\right) \\
& (A, \phi)=\underset{\iota \in I}{*}\left(A_{\iota}, \tilde{\phi}_{\iota} \uparrow_{A_{\iota}}\right)
\end{aligned}
$$

be the reduced amalgamated free products of $C^{*}$-algebras. Then there is a unique $*$-homomorphism $\kappa: A \rightarrow \widetilde{A}$ such that for every $\iota \in I$ the diagram

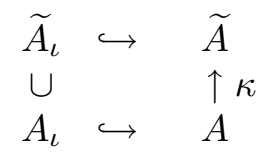


commutes, where the horizontal arrows are the inclusions arising from the free product construction. Moreover, $\kappa$ is necessarily injective.

Proof. Since $A$ is generated by $\bigcup_{\iota \in I} A_{\iota}$, it is clear that $\kappa$ will be unique if it exists. Let 1 denote the identity element of $\widetilde{B}$ and let $p$ be the identity element of $B$. If $p \neq 1$ then we may replace $B$ by $B+\mathbf{C}(1-p)$ and each $A_{\iota}$ by $A_{\iota}+\mathbf{C}(1-p)$; hence we may without loss of generality assume that $B$ is a unital $\mathrm{C}^{*}$-subalgebra of $\widetilde{B}$ and thus each $A_{\iota}$ is a unital $\mathrm{C}^{*}$-subalgebra of $\widetilde{A}_{\iota}$. Let

$$
\begin{aligned}
& \left(\widetilde{\pi}_{\iota}, \widetilde{E}_{\iota}, \tilde{\xi}_{\iota}\right)=\operatorname{GNS}\left(\widetilde{A}_{\iota}, \tilde{\phi}_{\iota}\right), \\
& \left(\pi_{\iota}, E_{\iota}, \xi_{\iota}\right)=\operatorname{GNS}\left(A_{\iota}, \phi_{\iota}\right)
\end{aligned}
$$

and

$$
\begin{aligned}
& (\widetilde{E}, \tilde{\xi})=\underset{\iota \in I}{*}\left(\widetilde{E}_{\iota}, \tilde{\xi}_{\iota}\right), \\
& (E, \xi)=\underset{\iota \in I}{*}\left(E_{\iota}, \xi_{\iota}\right) .
\end{aligned}
$$

The inclusion $A_{\iota} \hookrightarrow \widetilde{A}_{\iota}$ gives an inner-product-preserving isometry of Banach spaces $E_{\iota} \hookrightarrow \widetilde{E}_{\iota}$ sending $\xi_{\iota}$ to $\tilde{\xi}_{\iota}$, and we identify $E_{\iota}$ with this subspace of $\widetilde{E}_{\iota}$ and thereby $E_{\iota}^{\mathrm{o}}$ with the subspace of $\widetilde{E}_{\iota}^{\mathrm{o}}$. This allows canonical identification of the tensor product module

$$
E_{\iota_{1}}^{\mathrm{o}} \otimes_{B} \cdots \otimes_{B} E_{\iota_{p-1}}^{\mathrm{o}} \otimes_{B} \widetilde{E}_{\iota_{p}}^{\mathrm{o}} \otimes_{\widetilde{B}} \cdots \otimes_{\widetilde{B}} \widetilde{E}_{\iota_{n}}^{\mathrm{o}}
$$

with a closed subspace of $\widetilde{E}_{\iota_{1}}^{\mathrm{o}} \otimes_{\widetilde{B}} \cdots \otimes_{\widetilde{B}} \widetilde{E}_{\iota_{n}}^{\mathrm{o}}$. Hence, we may and do identify $E$ with the subspace

$$
\tilde{\xi} B \oplus \bigoplus_{\substack{n \geq 1 \\ \iota_{1}, \ldots, \iota_{n} \in I \\ \iota_{1} \neq \iota_{2}, \ldots, \iota_{n-1} \neq \iota_{n}}} E_{\iota_{1}}^{\mathrm{o}} \otimes_{B} \cdots \otimes_{B} E_{\iota_{n}}^{\mathrm{o}}
$$

of $\widetilde{E}$. Let $\mathfrak{A}=\left(*_{B}\right)_{\iota \in I} A_{\iota}$ be the universal algebraic free product with amalgamation over $B$. Let $\sigma: \mathfrak{A} \rightarrow \mathcal{L}(E)$, respectively $\widetilde{\sigma}: \mathfrak{A} \rightarrow \mathcal{L}(\widetilde{E})$, be the homomorphism extending the homomorphisms $\pi_{\iota}: A_{\iota} \rightarrow \mathcal{L}(E)$, respectively $\tilde{\pi}_{\iota} \uparrow_{A_{\iota}}: A_{\iota} \rightarrow \mathcal{L}(\widetilde{E}),(\iota \in I)$. In particular, we have $\overline{\sigma(\mathfrak{A})}=A$. In order to show that $\kappa$ exists, it will suffice to show that

$$
\forall x \in \mathfrak{A} \quad\|\widetilde{\sigma}(x)\| \leq\|\sigma(x)\| .
$$

Note that the subspace $E$ of $\widetilde{E}$ is invariant under $\widetilde{\sigma}(\mathfrak{A})$ and that the restriction of $\widetilde{\sigma}(\cdot)$ to $E$ gives $\sigma$. This implies

$$
\forall x \in \mathfrak{A} \quad\|\widetilde{\sigma}(x)\| \geq\|\sigma(x)\|,
$$

which will in turn imply that $\kappa$ is injective, once it is known to exist. Let $\tau$ be a faithful representation of $\widetilde{B}$ on a Hilbert space $\mathcal{W}$. Consider the Hilbert 
space $\widetilde{E} \otimes_{\tau} \mathcal{W}$ and let $\widetilde{\lambda}: \mathcal{L}(\widetilde{E}) \rightarrow \mathcal{L}\left(\widetilde{E} \otimes_{\tau} \mathcal{W}\right)$ be the $*$-homomorphism given by $\tilde{\lambda}(x)=x \otimes 1_{\mathcal{W}}$. Then $\tilde{\lambda}$ is faithful, and hence it will suffice to show that

$$
\forall x \in \mathfrak{A} \quad\|\widetilde{\lambda} \circ \widetilde{\sigma}(x)\| \leq\|\sigma(x)\| .
$$

Our strategy will be to show that $\widetilde{\lambda} \circ \widetilde{\sigma}$ decomposes as a direct sum of subrepresentations, each of which is of the form $\left(\nu l^{A}\right) \circ \sigma$, where $\nu l^{A}$ is the *-representation of $A$ induced from a representation $\nu$ of some $A_{\iota}$.

Given $n \geq 1$ and $\iota_{1}, \ldots, \iota_{n}$ with $\iota_{1} \neq \iota_{2}, \ldots, \iota_{n-1} \neq \iota_{n}$, and given $p \in$ $\{1,2, \ldots, n\}$, consider the Hilbert space

$$
\begin{aligned}
& E_{\iota_{1}}^{\mathrm{o}} \otimes_{B} \cdots \otimes_{B} E_{\iota_{p-1}}^{\mathrm{o}} \otimes_{B} K_{\iota_{p}} \otimes_{\widetilde{B}} \widetilde{E}_{\iota_{p+1}}^{\mathrm{o}} \otimes_{\widetilde{B}} \cdots \otimes_{\widetilde{B}} \widetilde{E}_{\iota_{n}}^{\mathrm{o}} \otimes_{\tau} \mathcal{W}= \\
& \quad \stackrel{\text { def }}{=}\left(\begin{array}{c}
E_{\iota_{1}}^{\mathrm{o}} \otimes_{B} \cdots \otimes_{B} E_{\iota_{p-1}}^{\mathrm{o}} \otimes_{B} \widetilde{E}_{\iota_{p}}^{\mathrm{o}} \otimes_{\widetilde{B}} \widetilde{E}_{\iota_{p+1}}^{\mathrm{o}} \otimes_{\widetilde{B}} \cdots \otimes_{\widetilde{B}} \widetilde{E}_{\iota_{n}}^{\mathrm{o}} \otimes_{\tau} \mathcal{W} \\
E_{\iota_{1}}^{\mathrm{o}} \otimes_{B} \cdots \otimes_{B} E_{\iota_{p-1}}^{\mathrm{o}} \otimes_{B} E_{\iota_{p}}^{\mathrm{o}} \otimes_{B} \widetilde{E}_{\iota_{p+1}}^{\mathrm{o}} \otimes_{\widetilde{B}} \cdots \otimes_{\widetilde{B}} \widetilde{E}_{\iota_{n}}^{\mathrm{o}} \otimes_{\tau} \mathcal{W}
\end{array}\right) .
\end{aligned}
$$

Heuristically, $K_{\iota}$ takes the place of $\widetilde{E}_{\iota} \ominus E_{\iota}$, even when the latter does not make sense. Then

$$
\begin{gathered}
\widetilde{E} \otimes_{\tau} \mathcal{W}=\left(E \otimes_{\tau \uparrow_{B}} \mathcal{W}\right) \oplus \\
\oplus \bigoplus_{\begin{array}{c}
n \geq 1 \\
\iota_{1}, \ldots, \iota_{n} \in I \\
\iota_{1} \neq \iota_{2}, \ldots, \iota_{n-1} \neq \iota_{n} \\
p \in\{1,2, \ldots, n\}
\end{array}} E_{\iota_{1}}^{\mathrm{o}} \otimes_{B} \cdots \otimes_{B} E_{B}^{\mathrm{o}} \otimes_{\iota_{p-1}} \otimes_{B} \\
\otimes_{\iota_{p}} \otimes_{\widetilde{B}} \widetilde{E}_{\iota_{p+1}}^{\mathrm{o}} \otimes_{\widetilde{B}} \cdots \otimes_{\widetilde{B}} \widetilde{E}_{\iota_{n}}^{\mathrm{o}} \otimes_{\tau} \mathcal{W} .
\end{gathered}
$$

As mentioned earlier, $\widetilde{\sigma}(\mathfrak{A}) E \subseteq E$ and $\widetilde{\sigma}(\cdot) \uparrow_{E}=\sigma(\cdot)$, so $E \otimes_{\tau \uparrow_{B}} \mathcal{W}$ is invariant under $\widetilde{\lambda} \circ \tilde{\sigma}(\mathfrak{A})$, and

$$
\forall x \in \mathfrak{A} \quad \| \widetilde{\lambda} \circ \tilde{\sigma}(x)\left\lceil_{E \otimes_{\tau} \mathcal{W}}\|=\| \sigma(x) \| .\right.
$$

Since $\tilde{\pi}_{\iota}\left(A_{\iota}\right) E_{\iota} \subseteq E_{\iota}$, it is not difficult to check that for every $n \geq 1$ and for every $\iota_{1}, \ldots, \iota_{n} \in I$ with $\iota_{1} \neq \iota_{2}, \ldots, \iota_{n-1} \neq \iota_{n}$,

$$
\begin{aligned}
& \widetilde{\mathcal{W}}\left(\iota_{1}, \ldots, \iota_{n}\right) \\
& \stackrel{\text { def }}{=} \widetilde{\widetilde{\lambda} \circ \widetilde{\sigma}(\mathfrak{A})\left(K_{\iota_{1}} \otimes_{\widetilde{B}} \widetilde{E}_{\iota_{2}}^{\mathrm{o}} \otimes_{\widetilde{B}} \cdots \otimes_{\widetilde{B}} \widetilde{E}_{\iota_{n}}^{\mathrm{o}} \otimes_{\tau} \mathcal{W}\right)} \\
& =\left(K_{\iota_{1}} \otimes_{\widetilde{B}} \widetilde{E}_{\iota_{2}}^{\mathrm{o}} \otimes_{\widetilde{B}} \cdots \otimes_{\widetilde{B}} \widetilde{E}_{\iota_{n}}^{\mathrm{o}} \otimes_{\tau} \mathcal{W}\right) \quad \oplus \\
& \oplus \bigoplus_{q>1} \quad E_{\iota_{1}^{\prime}}^{\mathrm{o}} \otimes_{B} \cdots \otimes_{B} E_{\iota_{q}^{\prime}}^{\mathrm{o}} \otimes_{B} K_{\iota_{1}} \otimes_{\widetilde{B}} \widetilde{E}_{\iota_{2}}^{\mathrm{o}} \otimes_{\widetilde{B}} \cdots \otimes_{\widetilde{B}} \widetilde{E}_{\iota_{n}}^{\mathrm{o}} \otimes_{\tau} \mathcal{W} \text {. } \\
& \begin{array}{c}
q \geq 1 \\
\iota_{1}^{\prime}, \ldots, \iota_{q}^{\prime} \in I \\
\iota_{1}^{\prime} \neq \iota_{2}^{\prime}, \ldots, \iota_{q-1}^{\prime} \neq \iota_{q}^{\prime} \\
\iota_{q}^{\prime} \neq \iota_{1}
\end{array}
\end{aligned}
$$

Thus

$$
\widetilde{E} \otimes_{\tau} \mathcal{W}=\left(E \otimes_{\tau \uparrow_{B}} \mathcal{W}\right) \oplus \bigoplus_{\substack{n \geq 1 \\ \iota_{1}, \ldots, \iota_{n} \in I \\ \iota_{1} \neq \iota_{2}, \ldots, \iota_{n-1} \neq \iota_{n}}} \tilde{\mathcal{W}}\left(\iota_{1}, \ldots, \iota_{n}\right) ;
$$


hence in order to prove the theorem it will suffice to show that for every choice of $\iota_{1}, \ldots \iota_{n}$,

$$
\forall x \in \mathfrak{A} \quad\left\|\widetilde{\lambda} \circ \tilde{\sigma}(x) \Gamma_{\tilde{\mathcal{W}}\left(\iota_{1}, \ldots, \iota_{n}\right)}\right\| \leq\|\sigma(x)\| .
$$

But letting $\mathcal{V}=K_{\iota_{1}} \otimes_{\widetilde{B}} \widetilde{E}_{\iota_{2}}^{\mathrm{o}} \otimes_{\widetilde{B}} \cdots \otimes_{\widetilde{B}} \widetilde{E}_{\iota_{n}}^{\mathrm{o}} \otimes_{\tau} \mathcal{W}$, letting $\nu: A_{\iota_{1}} \rightarrow \mathcal{L}(\mathcal{V})$ be the $*$-homomorphism $\nu(a)=\left(\widetilde{\pi}_{\iota_{1}}(a) \otimes 1_{\widetilde{E}_{\iota_{2}}^{\mathrm{o}} \otimes_{B} \cdots \otimes_{B} \widetilde{E}_{\iota_{n}} \otimes_{\tau} \mathcal{W}}\right) \Gamma_{\mathcal{V}}$, and appealing to Explication 1.2, it is straightforward to check that

$$
\widetilde{\lambda} \circ \widetilde{\sigma}(\cdot) \Gamma_{\widetilde{\mathcal{W}}\left(\iota_{1}, \ldots, \iota_{n}\right)}=\left(\nu \iota^{A}\right) \circ \sigma,
$$

where $\nu l^{A}$ is the representation of $A$ induced from $\nu$ with respect to the conditional expectation $\Phi_{\iota_{1}}: A \rightarrow A_{\iota_{1}}$ found in Lemma 1.1; this in turn implies (6).

Remark 1.4. Let us consider for a moment Theorem 1.3 when the subalgebra $B$ over which we amalgamate is the scalars, C. When taking the reduced free product $(A, \phi)=\underset{\iota \in I}{*}\left(A_{\iota}, \phi_{\iota}\right)$ of $\mathrm{C}^{*}$-algebras, one usually requires the states $\phi_{\iota}$ to have faithful GNS representation. However, one could extend the construction to the case of completely general states $\phi_{\iota}$; one then obtains

$$
\underset{\iota \in I}{*}\left(A_{\iota}, \phi_{\iota}\right)=\underset{\iota \in I}{*}\left(\left(A_{\iota} / \operatorname{ker} \pi_{\iota}\right), \dot{\phi}_{\iota}\right),
$$

where $\pi_{\iota}$ is the GNS representation of $\phi_{\iota}$ and where $\dot{\phi}_{\iota}$ is the state induced on the quotient $A_{\iota} / \operatorname{ker} \pi_{\iota}$ by $\phi_{\iota}$. Thus the canonical $*$-homomorphism $A_{\iota} \rightarrow A$ has the same kernel as $\pi_{\iota}$.

As a caveat, we would like to point out that with this relaxed definition of reduced free product, (allowing $\phi_{\iota}$ with nonfaithful GNS representation), the statement of Theorem 1.3 does not in general hold. Indeed, if for some $\iota \in I$ $A_{\iota}=\mathbf{C} \oplus \mathbf{C}$ with $\phi_{\iota}$ non-faithful, if $\widetilde{A}_{\iota}=M_{2}(\mathbf{C})$ with a unital embedding $A_{\iota} \hookrightarrow \widetilde{A}_{\iota}$ and if $\tilde{\phi}_{\iota}$ is a state on $M_{2}(\mathbf{C})$ such that $\tilde{\phi} \uparrow_{A_{\iota}}=\phi_{\iota}$, then $A_{\iota} \rightarrow A$ is not injective, while $\widetilde{A}_{\iota} \hookrightarrow \widetilde{A}$ is injective. This shows that there can be no *-homomorphism $\kappa: A \rightarrow \widetilde{A}$ making the diagram (4) commute. However, there is no problem allowing the $\tilde{\phi}_{\iota}$ to have nonfaithful GNS representations, as long as the restrictions $\phi_{\iota}$ are taken with faithful GNS representations.

\section{Completely positive maps.}

M. Choda [7] gave an argument which, when combined with an embedding result like Property 2, proves that if $\theta_{\iota}: A_{\iota} \rightarrow D_{\iota}$ is a unital completely positive map between unital $\mathrm{C}^{*}$-algebras for every $\iota \in I$, if $\phi_{\iota}$ and $\psi_{\iota}$ are states on $A_{\iota}$ and respectively $D_{\iota}$, each having faithful GNS representation, 
and if $\psi_{\iota} \circ \theta_{\iota}=\phi_{\iota}$ then letting

$$
\begin{aligned}
(A, \phi) & =\underset{\iota \in I}{*}\left(A_{\iota}, \phi_{\iota}\right) \\
(D, \psi) & =\underset{\iota \in I}{*}\left(D_{\iota}, \psi_{\iota}\right)
\end{aligned}
$$

be the reduced free products of $\mathrm{C}^{*}$-algebras, there is a unital completely positive map $\theta: A \rightarrow D$ such that $\theta \uparrow_{A_{\iota}}=\theta_{\iota}$ for every $\iota \in I$, and such that $\theta\left(a_{1} a_{2} \cdots a_{n}\right)=\theta\left(a_{1}\right) \theta\left(a_{2}\right) \cdots \theta\left(a_{n}\right)$ whenever $a_{j} \in A_{\iota_{j}} \cap \operatorname{ker} \phi_{\iota_{j}}$ for some $\iota_{j} \in I$ with $\iota_{1} \neq \iota_{2}, \ldots, \iota_{n-1} \neq \iota_{n}$.

In this section, we generalize this argument of Choda's to the case of reduced amalgamated free products of $\mathrm{C}^{*}$-algebras. The generalization consists of, in essence, replacing Stinespring's dilation theorem for completely positive maps into bounded operators on a Hilbert space by Kasparov's generalization [17] to the case of completely positive maps into the algebra of bounded adjointable operators on a Hilbert $B$-module (see alternatively the book [18]). We would like to point out that Theorem 2.2 is quite similar in appearance to analogous results of $\mathrm{F}$. Boca [2], [3] about completely positive maps on universal amalgamated free products of $\mathrm{C}^{*}$-algebras. However, the universal and reduced free products of $\mathrm{C}^{*}$-algebras are quite different in character, and we do not believe that Boca's results can be used directly to prove Theorem 2.2 .

Lemma 2.1. Let $A$ and $B$ be $C^{*}$-algebras, let $E$ and $\widetilde{E}$ be Hilbert $A$-modules, let $F$ and $\tilde{F}$ be Hilbert $B$-modules and let $v \in \mathcal{L}(E, \widetilde{E}), w \in \mathcal{L}(F, \tilde{F})$. Suppose $\pi: A \rightarrow \mathcal{L}(F)$ and $\tilde{\pi}: A \rightarrow \mathcal{L}(\tilde{F})$ are $*$-homomorphisms and suppose that

$$
\forall a \in A \quad \forall \xi \in F \quad w(\pi(a) \xi)=\widetilde{\pi}(a) w(\xi) .
$$

Let $E \otimes_{\pi} F$ and $\widetilde{E} \otimes_{\tilde{\pi}} \tilde{F}$ be the interior tensor products. Then there is an element $v \otimes w \in \mathcal{L}\left(E \otimes_{\pi} F, \widetilde{E} \otimes_{\tilde{\pi}} \tilde{F}\right)$ such that

$$
\forall \zeta \in E \quad \forall \xi \in F \quad(v \otimes w)(\zeta \otimes \xi)=(v \zeta) \otimes(w \xi) .
$$

If, moreover, $\langle v(\zeta), v(\zeta)\rangle=\langle\zeta, \zeta\rangle$ for every $\zeta \in E$ and $\langle w(\xi), w(\xi)\rangle=\langle\xi, \xi\rangle$ for every $\xi \in F$ then $\langle v \otimes w(\eta), v \otimes w(\eta)\rangle=\langle\eta, \eta\rangle$ for every $\eta \in E \otimes_{\pi} F$.

Proof. That $v \otimes w$ is bounded is a standard argument (compare p. 42 of [18]). Then one sees $(v \otimes w)^{*}=v^{*} \otimes w^{*}$. The final statement follows using the polarization identity.

Theorem 2.2. Let $B$ be a unital $C^{*}$-algebra, let $I$ be a set and for every $\iota \in I$ let $A_{\iota}$ and $D_{\iota}$ be unital $C^{*}$-algebras containing copies of $B$ as unital $C^{*}$-subalgebras and having conditional expectations $\phi_{\iota}: A_{\iota} \rightarrow B$, respectively $\psi_{\iota}: D_{\iota} \rightarrow B$, whose GNS representations are faithful. Suppose that for each $\iota \in I$ there is a unital completely positive map $\theta_{\iota}: A_{\iota} \rightarrow D_{\iota}$ that is also a 
$B-B$ bimodule map and satisfies $\psi_{\iota} \circ \theta_{\iota}=\phi_{\iota}$. Let

$$
\begin{aligned}
& (A, \phi)=\underset{\iota \in I}{*}\left(A_{\iota}, \phi_{\iota}\right) \\
& (D, \phi)=\underset{\iota \in I}{*}\left(D_{\iota}, \psi_{\iota}\right)
\end{aligned}
$$

be the reduced amalgamated free products of $C^{*}$-algebras. Then there is a unital completely positive map $\theta: A \rightarrow D$ such that for all $\iota \in I$ the diagram

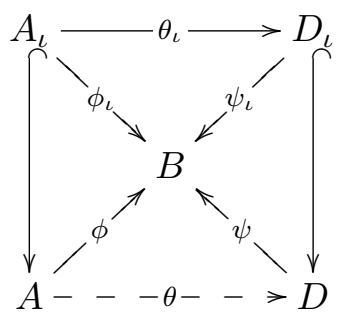

commutes, where the vertical inclusions are those arising from the free product construction, and satisfying

$$
\theta\left(a_{1} a_{2} \cdots a_{n}\right)=\theta\left(a_{1}\right) \theta\left(a_{2}\right) \cdots \theta\left(a_{n}\right)
$$

whenever $a_{j} \in A_{\iota_{j}} \cap \operatorname{ker} \phi_{\iota_{j}}$ and $\iota_{1} \neq \iota_{2}, \iota_{2} \neq \iota_{3}, \ldots, \iota_{n-1} \neq \iota_{n}$.

Proof. Note first that the assumptions imply that each $\theta_{\iota}$ is the identity map on $B$. Let

$$
\left(\pi_{\iota}, E_{\iota}, \xi_{\iota}\right)=\operatorname{GNS}\left(D_{\iota}, \psi_{\iota}\right), \quad(E, \xi)=\underset{\iota \in I}{*}\left(E_{\iota}, \xi_{\iota}\right) .
$$

(We will usually write simply $d \zeta$ instead of $\pi_{\iota}(d) \zeta$, when $d \in D_{\iota}$ and $\zeta \in E_{\iota}$.) Recall that then $E_{\iota}=\xi_{\iota} B \oplus E_{\iota}^{\mathrm{o}}$, that the action $\left.\pi_{\iota}\right|_{B}$ leaves $E_{\iota}^{\mathrm{o}}$ globally invariant, and that

$$
E=\xi B \oplus \bigoplus_{\substack{n \geq 1 \\ \iota_{1}, \ldots, \iota_{n} \in I \\ \iota_{1} \neq \iota_{2}, \ldots, \iota_{n-1} \neq \iota_{n}}} E_{\iota_{1}}^{\mathrm{o}} \otimes_{B} \cdots \otimes_{B} E_{\iota_{n}}^{\mathrm{o}} .
$$

Consider the Hilbert $B$-module $F_{\iota}=A_{\iota} \otimes_{\pi_{\iota} \circ \theta_{\iota}} E_{\iota}$ and the specified element $\eta_{\iota}=1 \otimes \xi_{\iota} \in F_{\iota}$. Since $\theta_{\iota}$ restricts to the identity map on $B$, in $F_{\iota}$ we have $b \otimes \zeta=1 \otimes(b \zeta)$ for every $b \in B$ and $\zeta \in E$. Consider the unital *-homomorphism $\sigma_{\iota}: A_{\iota} \rightarrow \mathcal{L}\left(F_{\iota}\right)$ given by

$$
\forall a^{\prime}, a \in A_{\iota} \quad \forall \zeta \in E_{\iota} \quad \sigma_{\iota}\left(a^{\prime}\right)(a \otimes \zeta)=\left(a^{\prime} a\right) \otimes \zeta,
$$

(cf. page 48 of [18]). Consider the map $\rho_{\iota}: \mathcal{L}\left(F_{\iota}\right) \rightarrow B$ given by $\rho_{\iota}(x)=$ $\left\langle\eta_{\iota}, x \eta_{\iota}\right\rangle$. If $x \in \mathcal{L}\left(F_{\iota}\right)$ and if $b_{1}, b_{2} \in B$ then $\rho_{\iota}\left(\sigma_{\iota}\left(b_{1}\right) x \sigma_{\iota}\left(b_{2}\right)\right)=b_{1} \rho_{\iota}(x) b_{2}$. If we use $\sigma_{\iota}$ to identify $B$ with $\sigma_{\iota}(B) \subseteq \mathcal{L}\left(F_{\iota}\right)$ then we have that $\rho_{\iota}: \mathcal{L}\left(F_{\iota}\right) \rightarrow$ $B$ is a conditional expectation. Clearly $L^{2}\left(\mathcal{L}\left(F_{\iota}\right), \rho_{\iota}\right) \cong F_{\iota}$ and the GNS representation of $\rho_{\iota}$ is faithful on $\mathcal{L}\left(F_{\iota}\right)$. We have that $\rho_{\iota} \circ \sigma_{\iota}=\phi_{\iota}$ since

$$
\rho_{\iota} \circ \sigma_{\iota}(a)=\left\langle 1 \otimes \xi_{\iota}, a \otimes \xi_{\iota}\right\rangle=\left\langle\xi_{\iota}, \theta_{\iota}(a) \xi_{\iota}\right\rangle=\psi_{\iota} \circ \theta_{\iota}(a)=\phi_{\iota}(a) .
$$


Let

$$
(\mathcal{M}, \rho)=\underset{\iota \in I}{*}\left(\mathcal{L}\left(F_{\iota}\right), \rho_{\iota}\right)
$$

be the reduced amalgamated free product of $\mathrm{C}^{*}$-algebras. Note that $\mathcal{M} \subseteq$ $\mathcal{L}(F)$ where

$$
(F, \eta)=\underset{\iota \in I}{*}\left(F_{\iota}, \eta_{\iota}\right)
$$

By Theorem 1.3 there is a $*$-homomorphism $\sigma: A \rightarrow \mathcal{M}$ such that $\left.\sigma\right|_{A_{\iota}}=\sigma_{\iota}$, $(\iota \in I)$.

Consider the operator $v_{\iota}: E_{\iota} \rightarrow F_{\iota}$ given by $\zeta \rightarrow 1 \otimes \zeta$, and note that $\left\langle v_{\iota} \zeta, v_{\iota} \zeta\right\rangle=\langle\zeta, \zeta\rangle$ for every $\zeta \in E_{\iota}$, hence $v_{\iota}\left(E_{\iota}^{\mathrm{o}}\right) \subseteq F_{\iota}^{\mathrm{o}}$. A calculation using e.g. Lemma 5.4 of [18] shows that there is a bounded operator $F_{\iota} \rightarrow E_{\iota}$ sending $a \otimes \zeta$ to $\theta_{\iota}(a) \zeta$, which is then the adjoint of $v_{\iota}$. Hence $v_{\iota} \in \mathcal{L}\left(E_{\iota}, F_{\iota}\right)$, and clearly $v_{\iota}^{*} v_{\iota}=1$. Since $\theta_{\iota}$ is a left $B$-module map, we have for every $b \in B$ and $\zeta \in E_{\iota}$ that $v_{\iota}(b \zeta)=1 \otimes(b \zeta)=b \otimes \zeta=b\left(v_{\iota}(\zeta)\right)$. Therefore, taking direct sums of operators $v_{\iota_{1}} \otimes \cdots \otimes v_{\iota_{n}}$ given by Lemma 2.1, we get $v \in \mathcal{L}(E, F)$ such that $\langle v \zeta, v \zeta\rangle=\langle\zeta, \zeta\rangle$ for every $\zeta \in E, v \xi=\eta$ and

$$
v\left(\zeta_{1} \otimes \zeta_{2} \otimes \cdots \otimes \zeta_{n}\right)=\left(v_{\iota_{1}} \zeta_{1}\right) \otimes\left(v_{\iota_{2}} \zeta_{2}\right) \otimes \cdots \otimes\left(v_{\iota_{n}} \zeta_{n}\right)
$$

whenever $\zeta_{j} \in E_{\iota_{j}}^{\mathrm{o}}, \iota_{1}, \ldots, \iota_{n} \in I$ and $\iota_{j} \neq \iota_{j+1}$. Let $\theta: A \rightarrow \mathcal{L}(E)$ be the unital completely positive map $\theta(x)=v^{*} \sigma(x) v$.

We will show that the diagram (8) commutes and and that (9) holds, which will furthermore imply that $\theta(A) \subseteq D$. In order to show (8) commutes, let $w_{\iota}: E \rightarrow E_{\iota} \otimes_{B} E(\iota)$ and $y_{\iota}: F \rightarrow F_{\iota} \otimes_{B} F(\iota)$ be the unitaries used in the free product constructions to define the inclusions $A_{\iota} \hookrightarrow A$ and, respectively, $\mathcal{L}\left(F_{\iota}\right) \hookrightarrow \mathcal{M}$. Note that $v_{\iota}(E(\iota)) \subseteq F(\iota)$ and that $y_{\iota} v=\left(v_{\iota} \otimes v \uparrow_{E(\iota)}\right) w_{\iota}$. Furthermore, observe that for $a \in A_{\iota}$ and $\zeta \in E_{\iota}$,

$$
\left(v_{\iota}^{*} \sigma_{\iota}(a) v_{\iota}\right) \zeta=v_{\iota}^{*}(a \otimes \zeta)=\theta_{\iota}(a) \zeta .
$$

Hence for $a \in A_{\iota}$,

$$
\begin{aligned}
\theta(a) & =v^{*} \sigma(a) v=v^{*} \sigma_{\iota}(a) v=v^{*} y_{\iota}^{*}\left(\sigma_{\iota}(a) \otimes 1_{F(\iota)}\right) y_{\iota} v= \\
& =w_{\iota}^{*}\left(v_{\iota}^{*} \sigma_{\iota}(a) v_{\iota} \otimes\left(v \uparrow_{E(\iota)}\right)^{*} v \uparrow_{E(\iota)}\right) w_{\iota}=w_{\iota}^{*}\left(\theta_{\iota}(a) \otimes 1_{E(\iota)}\right) w_{\iota}=\theta_{\iota}(a),
\end{aligned}
$$

and thus (8) commutes. Now to show that (9) holds, consider $a_{j} \in A_{\iota_{j}} \cap$ $\operatorname{ker} \phi_{\iota_{j}}$ for some $\iota_{j} \in I(1 \leq j \leq n)$ with $\iota_{j} \neq \iota_{j+1}$. It is easy to see that

$$
\begin{aligned}
\theta_{\iota_{1}}\left(a_{1}\right) \cdots \theta_{\iota_{n}}\left(a_{n}\right) \xi & =\widehat{\theta_{\iota_{1}}\left(a_{1}\right)} \otimes \cdots \otimes \widehat{\theta_{\iota_{n}}\left(a_{n}\right)} \\
& =v^{*}\left(\left(a_{1} \otimes \xi_{\iota_{1}}\right) \otimes \cdots \otimes\left(a_{n} \otimes \xi_{\iota_{n}}\right)\right)=\theta\left(a_{1} \cdots a_{n}\right) \xi
\end{aligned}
$$

Now consider an element $\zeta_{1} \otimes \cdots \otimes \zeta_{p} \in E$, where $\zeta_{j} \in E_{k_{j}}^{\mathrm{o}}$ for some $k_{j} \in I$ with $k_{j} \neq k_{j+1}$.

Let $P_{0}: E \rightarrow \xi B$ be the projection and for $\ell \in \mathbf{N}$ let

$$
P_{\ell}: E \rightarrow \bigoplus_{\substack{\iota_{1}, \ldots, \iota_{\ell} \in I \\ \iota_{1} \neq \iota_{2}, \ldots, \iota_{\ell-1} \neq \iota_{\ell}}} E_{\iota_{1}}^{\mathrm{o}} \otimes \cdots \otimes E_{\iota_{\ell}}
$$


be the projection. Taking adjoints and using (11), we see that

$$
P_{0} \theta_{\iota_{1}}\left(a_{1}\right) \cdots \theta_{\iota_{n}}\left(a_{n}\right)\left(\zeta_{1} \otimes \cdots \otimes \zeta_{p}\right)=P_{0} \theta\left(a_{1} \cdots a_{n}\right)\left(\zeta_{1} \otimes \cdots \otimes \zeta_{p}\right) .
$$

Now letting $\ell \in \mathbf{N}$ we will use standard techniques (see, for example, [14] and $[\mathbf{1 2}])$ to show that

$$
P_{\ell} \theta_{\iota_{1}}\left(a_{1}\right) \cdots \theta_{\iota_{n}}\left(a_{n}\right)\left(\zeta_{1} \otimes \cdots \otimes \zeta_{p}\right)=P_{\ell} \theta\left(a_{1} \cdots a_{n}\right)\left(\zeta_{1} \otimes \cdots \otimes \zeta_{p}\right) .
$$

If $\ell>n+p$ or $\ell<|n-p|$ then it is clear that both sides of (12) are zero. If $\ell=n+p$ then both sides of (12) are zero unless $\iota_{n} \neq k_{1}$, in which case a calculation similar to (11) shows that (12) holds. Let $Q_{\iota}^{\mathrm{o}}: E_{\iota} \rightarrow E_{\iota}^{\mathrm{o}}$ and $R_{\iota}^{\mathrm{o}}: F_{\iota} \rightarrow F_{\iota}^{\mathrm{o}}$ be the projections, and note that $R_{\iota}^{\mathrm{o}} v_{\iota}=v_{\iota} Q_{\iota}^{\mathrm{o}}$. Consider when $n+p-\ell=1$. Then both sides of (12) are zero unless $\iota_{n}=k_{1}$, in which case

$$
\begin{aligned}
& P_{\ell} \theta_{\iota_{1}}\left(a_{1}\right) \cdots \theta_{\iota_{n}}\left(a_{n}\right)\left(\zeta_{1} \otimes \cdots \otimes \zeta_{p}\right) \\
& =\widehat{\theta_{\iota_{1}}\left(a_{1}\right)} \otimes \cdots \otimes \theta_{\iota_{n-1}}\left(a_{n-1}\right) \otimes Q_{\iota_{n}}^{\mathrm{o}}\left(\theta_{\iota_{n}}\left(a_{n}\right) \zeta_{1}\right) \otimes \zeta_{2} \otimes \cdots \otimes \zeta_{p} \\
& =v_{\iota}^{*}\left(\left(a_{1} \otimes \xi_{\iota_{1}}\right) \otimes \cdots \otimes\left(a_{n-1} \otimes \xi_{\iota_{n-1}}\right) \otimes R_{\iota_{n}}^{\mathrm{o}}\left(a_{n} \otimes \zeta_{1}\right)\right. \\
& \left.\quad \otimes\left(1 \otimes \zeta_{2}\right) \otimes \cdots \otimes\left(1 \otimes \zeta_{p}\right)\right) \\
& =P_{\ell} \theta\left(a_{1} \cdots a_{n}\right)\left(\zeta_{1} \otimes \cdots \otimes \zeta_{p}\right) .
\end{aligned}
$$

If $p+n-\ell=2 r+1$ for $r \in\{1,2, \ldots, \min (p, n)-2\}$ then both sides of (12) are zero unless $\iota_{n}=k_{1}, \iota_{n-1}=k_{2}, \ldots, \iota_{n-r+1}=k_{r}$, in which case

$$
\begin{aligned}
P_{\ell} & \theta_{\iota_{1}}\left(a_{1}\right) \cdots \theta_{\iota_{n}}\left(a_{n}\right)\left(\zeta_{1} \otimes \cdots \otimes \zeta_{p}\right) \\
= & \widehat{\theta_{\iota_{1}}\left(a_{1}\right)} \otimes \cdots \otimes \theta_{\iota_{n-r-1}}\left(a_{n-r-1}\right) \otimes \\
& \otimes Q_{\iota_{n-r}}^{\mathrm{o}}\left(\theta_{\iota_{n-r}}\left(a_{n-r}\right)\left\langle\xi, \theta_{\iota_{n-r+1}}\left(a_{n-r+1}\right) \cdots \theta_{\iota_{n}}\left(a_{n}\right) \zeta_{1} \otimes \cdots \otimes \zeta_{r}\right\rangle \zeta_{r+1}\right) \otimes \\
& \otimes \zeta_{r+2} \otimes \cdots \otimes \zeta_{p} \\
= & \widehat{\theta_{\iota_{1}}\left(a_{1}\right)} \otimes \cdots \otimes \theta_{\iota_{n-r-1}}\left(\widehat{\left(a_{n-r-1}\right.}\right) \otimes \\
& \left.\otimes Q_{\iota_{n-r}}^{\mathrm{o}}\left(\theta_{\iota_{n-r}}\left(a_{n-r}\right)\left\langle\widehat{\theta_{\iota_{n}}\left(a_{n}^{*}\right)} \otimes \cdots \otimes \theta_{\iota_{n-r+1}\left(a_{n-r+1}^{*}\right.}\right), \zeta_{1} \otimes \cdots \otimes \zeta_{r}\right\rangle \zeta_{r+1}\right) \\
& \otimes \zeta_{r+2} \otimes \cdots \otimes \zeta_{p} \\
= & v^{*}\left(\left(a_{1} \otimes \xi_{\iota_{1}}\right) \otimes \cdots \otimes\left(a_{n-r-1} \otimes \xi_{\iota_{n-r-1}}\right) \otimes\right. \\
& \otimes R_{\iota_{n-r}}^{\mathrm{o}}\left(\theta_{\iota_{n-r}}\left(a_{n-r}\right) \cdot\right. \\
& \left.\cdot\left\langle\sigma_{\iota_{n}}\left(a_{n}^{*}\right) \cdots \sigma_{\iota_{n-r+1}}\left(a_{n-r+1}^{*}\right) \eta,\left(1 \otimes \zeta_{1}\right) \otimes \cdots \otimes\left(1 \otimes \zeta_{r}\right)\right\rangle\left(1 \otimes \zeta_{r+1}\right)\right) \otimes \\
& \left.\otimes\left(1 \otimes \zeta_{r+2}\right) \otimes \cdots \otimes\left(1 \otimes \zeta_{p}\right)\right)
\end{aligned}
$$




$$
\begin{aligned}
= & v^{*}\left(\left(a_{1} \otimes \xi_{\iota_{1}}\right) \otimes \cdots \otimes\left(a_{n-r-1} \otimes \xi_{\iota_{n-r-1}}\right) \otimes\right. \\
& \otimes R_{\iota_{n-r}}^{\mathrm{o}}\left(\theta_{\iota_{n-r}}\left(a_{n-r}\right) \cdot\right. \\
& \left.\cdot\left\langle\eta, \sigma_{\iota_{n-r+1}}\left(a_{n-r+1}\right) \cdots \sigma_{\iota_{n}}\left(a_{n}\right)\left(1 \otimes \zeta_{1}\right) \otimes \cdots \otimes\left(1 \otimes \zeta_{r}\right)\right\rangle\left(1 \otimes \zeta_{r+1}\right)\right) \otimes \\
& \left.\otimes\left(1 \otimes \zeta_{r+2}\right) \otimes \cdots \otimes\left(1 \otimes \zeta_{p}\right)\right) \\
= & P_{\ell} \theta\left(a_{1} \cdots a_{n}\right)\left(\zeta_{1} \otimes \cdots \otimes \zeta_{p}\right) .
\end{aligned}
$$

A similar calculation shows that (12) holds also when $n+p-\ell=2 \min (p, n)-$ 1.

If $n+p-\ell=2 r$ is even for $r \in\{1,2, \ldots, \min (p, n)-1\}$ then both sides of (12) are zero unless $\iota_{n}=k_{1}, \iota_{n-1}=k_{2}, \ldots, \iota_{n-r+1}=k_{r}$ and $\iota_{n-r} \neq k_{r+1}$, in which case

$$
\begin{aligned}
& P_{\ell} \theta_{\iota_{1}}\left(a_{1}\right) \cdots \theta_{\iota_{n}}\left(a_{n}\right)\left(\zeta_{1} \otimes \cdots \otimes \zeta_{p}\right) \\
& =\widehat{\theta_{\iota_{1}}\left(a_{1}\right)} \otimes \cdots \otimes \theta_{\iota_{n-r}\left(a_{n-r}\right)} \otimes \\
& \otimes\left\langle\xi, \theta_{\iota_{n-r+1}}\left(a_{n-r+1}\right) \cdots \theta_{\iota_{n}}\left(a_{n}\right) \zeta_{1} \otimes \cdots \otimes \zeta_{r}\right\rangle \zeta_{r+1} \otimes \\
& \otimes \zeta_{r+2} \otimes \cdots \otimes \zeta_{p} \\
& =\widehat{\theta_{\iota_{1}}\left(a_{1}\right)} \otimes \cdots \otimes \theta_{\iota_{n-r}\left(a_{n-r}\right)} \otimes \\
& \left.\otimes\left\langle\widehat{\left\langle\iota_{\iota_{n}}\left(a_{n}^{*}\right)\right.} \otimes \cdots \otimes \theta_{\iota_{n-r+1}} \widehat{\left(a_{n-r+1}^{*}\right.}\right), \zeta_{1} \otimes \cdots \otimes \zeta_{r}\right\rangle \zeta_{r+1} \otimes \\
& \otimes \zeta_{r+2} \otimes \cdots \otimes \zeta_{p} \\
& =v^{*}\left(\left(a_{1} \otimes \xi_{\iota_{1}}\right) \otimes \cdots \otimes\left(a_{n-r} \otimes \xi_{\iota_{n-r}}\right) \otimes\right. \\
& \otimes\left\langle\eta, \sigma_{\iota_{n-r+1}}\left(a_{n-r+1}\right) \cdots \sigma_{\iota_{n}}\left(a_{n}\right)\left(\left(1 \otimes \zeta_{1}\right) \otimes \cdots \otimes\left(1 \otimes \zeta_{r}\right)\right)\right\rangle\left(1 \otimes \zeta_{r+1}\right) \otimes \\
& \left.\otimes\left(1 \otimes \zeta_{r+2}\right) \otimes \cdots \otimes\left(1 \otimes \zeta_{p}\right)\right) \\
& =P_{\ell} \theta\left(a_{1} \cdots a_{n}\right)\left(\zeta_{1} \otimes \cdots \otimes \zeta_{p}\right) \text {. }
\end{aligned}
$$

Similar calculations show that (12) holds also when $p+n-\ell=2 \min (p, n)$. This finishes the proof of (9), and of the theorem.

\section{Amalgamated free products of von Neumann algebras.}

This section contains results for amalgamated free products of von Neumann algebras that are analogous to those for $\mathrm{C}^{*}$-algebras found in $\S 1$ and $\S 2$. The free product of von Neumann algebras with respect to given normal states was defined by Voiculescu in [21] and has been much studied. See also Ching's paper [6], where the free product of von Neumann algebras with respect to normal faithful tracial states from a certain class was first 
defined. We begin this section by describing the "folklore" construction of amalgamated free products of von Neumann algebras (with respect to normal conditional expectations onto a von Neumann subalgebra). We are grateful to the referee for pointing out this simplification of the construction we originally gave.

Construction 3.1. Let $B$ be a von Neumann algebra contained as a unital von Neumann subalgebra of von Neumann algebras $A_{\iota}(\iota \in I)$. Suppose there are normal conditional expectations $\phi_{\iota}: A_{\iota} \rightarrow B$. The amalgamated free product of von Neumann algebras, which we will denote

$$
(A, \phi)=\underset{\iota \in I}{\bar{*}}\left(A_{\iota}, \phi_{\iota}\right),
$$

is constructed as follows. Let

$$
(\mathcal{A}, \varphi)=\underset{\iota \in I}{*}\left(A_{\iota}, \phi_{\iota}\right)
$$

be the $\mathrm{C}^{*}$-algebra reduced amalgamated free product. Let $\psi$ be a normal state on $B$ with faithful GNS representation and consider the state $\psi \circ \varphi$ on $\mathcal{A}$. Let $\pi_{\psi \circ \varphi}$ be the GNS representation of $\mathcal{A}$ associated to $\psi \circ \varphi$, let $A=\pi_{\psi \circ \varphi}(\mathcal{A})^{\prime \prime} \subseteq \mathcal{L}\left(L^{2}(\mathcal{A}, \psi \circ \varphi)\right)$ and thereby regard $\mathcal{A}$ as a weakly dense subalgebra of $A$. The Hilbert space projection $L^{2}(\mathcal{A}, \psi \circ \varphi) \rightarrow L^{2}(B, \psi)$ gives rise to a normal conditional expectation $\phi: A \rightarrow B$ whose restriction to $\mathcal{A}$ is $\varphi$. It remains to see that the pair $(A, \phi)$ is independent of the choice of $\psi$. If $\psi^{\prime}$ is any normal state on $B$ then the state $\psi^{\prime} \circ \varphi$ of $\mathcal{A}$ extends to the normal state $\psi^{\prime} \circ \phi$ of $A$. Hence $\pi_{\psi^{\prime} \circ \varphi}(\mathcal{A})^{\prime \prime}=\pi_{\psi^{\prime} \circ \phi}(A)$ is a quotient of $A$. Thus $(A, \phi)$ is independent of the choice of $\psi$.

Remark 3.2. In the above construction, we have $\mathcal{A} \subseteq \mathcal{L}(E)$ for the Hilbert $B, B$-bimodule $E=L^{2}(\mathcal{A}, \varphi)$. The GNS Hilbert space $L^{2}(\mathcal{A}, \psi \circ \varphi)$ is canonically isomorphic to the internal tensor product $E \otimes_{\pi_{\psi}} L^{2}(B, \psi)$, where $\pi_{\psi}: B \rightarrow \mathcal{L}\left(L^{2}(B, \psi)\right)$ is the GNS representation of $\psi$, and the representation $\pi_{\psi \circ \varphi}$ is given by $\pi_{\psi \circ \varphi}(a)=a \otimes 1 \in \mathcal{L}\left(E \otimes_{\pi_{\psi}} L^{2}(B, \psi)\right)$. We will later use this picture in proofs.

Following Rieffel $[\mathbf{2 0}, 1.5]$, if $A$ and $B$ are von Neumann algebras, if $E$ is a Hilbert $B$-module and if $\theta: A \rightarrow \mathcal{L}(E)$ is a completely positive map, we say that $\theta$ is normal if for every $\zeta_{1}, \zeta_{2} \in E$, the map $A \ni a \mapsto$ $\left\langle\zeta_{1}, \theta(a) \zeta_{2}\right\rangle \in B$ is normal. This coincides with the usual notion of normality when $B=\mathbf{C}$ (in which case $E$ is a Hilbert space). It is clear that if $B$ is a von Neumann subalgebra of a von Neumann algebra $A$ having a normal conditional expectation $\phi: A \rightarrow B$ then the GNS representation of $A$ as bounded adjointable operators on the Hilbert $B$-module $L^{2}(A, \phi)$ is normal.

Part (i) of the following straightforward lemma was proved in the case of a $*$-homomorphism by Rieffel as part of $[\mathbf{2 0}, 5.2]$. 
Lemma 3.3. Let $A$ and $B$ be von Neumann algebras, let $E$ be a Hilbert $B$-module and suppose that $\theta: A \rightarrow \mathcal{L}(E)$ is completely positive map. Let $\mathcal{H}$ be a Hilbert space and let $\tau: B \rightarrow \mathcal{L}(\mathcal{H})$ be a normal $*$-representation. Let $\theta \otimes 1$ denote the completely positive map $A \ni a \mapsto \pi(a) \otimes 1 \in \mathcal{L}\left(E \otimes_{\tau} \mathcal{H}\right)$ of $A$ into bounded operators on the Hilbert space $E \otimes_{\tau} \mathcal{H}$. We have:

(i) if $\theta$ is normal then $\theta \otimes 1$ is normal;

(ii) if $\theta \otimes 1$ is normal and if $\tau$ is faithful then $\theta$ is normal.

Proof. If $\zeta_{1}, \zeta_{2} \in E$ and $v_{1}, v_{2} \in \mathcal{H}$ then

$$
\left\langle\zeta_{1} \otimes v_{1},(\theta \otimes 1)(x)\left(\zeta_{2} \otimes v_{2}\right)\right\rangle=\left\langle v_{1}, \tau\left(\left\langle\zeta_{1}, \theta(x) \zeta_{2}\right\rangle\right) v_{2}\right\rangle .
$$

If $\theta$ is normal then (13) shows that $\theta \otimes 1$ is continuous from the $\sigma\left(A, A_{*}\right)$ topology on $A$ to the weak-operator topology on $\mathcal{L}\left(E \otimes_{\tau} \mathcal{H}\right)$, which implies $\theta \otimes 1$ is normal and proves (i).

If $\theta \otimes 1$ is normal then (13) shows that $x \mapsto \tau\left(\left\langle\zeta_{1}, \theta(x) \zeta_{2}\right\rangle\right)$ is normal. Assuming also $\tau$ is faithful, it follows that $\theta$ is normal.

The following application of Lemma 3.3(i) is in $[\mathbf{2 0}, 5.2]$.

Lemma 3.4. Let $B$ be a unital von Neumann subalgebra of a von Neumann algebra $A$ with a normal conditional expectation $\Phi: A \rightarrow B$. Let $\tau$ be a normal *-representation of $B$ on a Hilbert space $\mathcal{H}$. Then the induced representation, $\tau l^{A}$, of $\tau$ to $A$ with respect to the conditional expectation $\Phi$ is normal.

Proof. By definition, and in the notation of Lemma 3.3, $\left.\tau\right|^{A}=\pi \otimes 1: A \rightarrow$ $\mathcal{L}\left(L^{2}(A, \Phi) \otimes_{\tau} \mathcal{H}\right)$, where $\pi$ is the GNS representation of $A$ on $L^{2}(A, \Phi)$.

Lemma 3.5. Let $A, B_{1}$ and $B_{2}$ be von Neumann algebras and let $E_{j}$ be a Hilbert $B_{j}$-module $(j=1,2)$. If $\theta: A \rightarrow \mathcal{L}\left(E_{1}\right)$ and $\sigma: B_{1} \rightarrow \mathcal{L}\left(E_{2}\right)$ are normal completely positive maps, then the completely positive map $\theta \otimes 1$ : $A \rightarrow \mathcal{L}\left(E_{1} \otimes_{\sigma} E_{2}\right)$ is normal.

Proof. Let $\tau: B_{2} \rightarrow \mathcal{L}(\mathcal{H})$ be a faithful normal $*$-representation of $B_{2}$ on a Hilbert space $\mathcal{H}$. Applying Lemma 3.3 in succession we find that $\sigma \otimes 1$ : $B_{1} \rightarrow \mathcal{L}\left(E_{2} \otimes_{\tau} \mathcal{H}\right)$ is normal, $\theta \otimes 1 \otimes 1: A \rightarrow \mathcal{L}\left(E_{1} \otimes_{\sigma} E_{2} \otimes_{\tau} \mathcal{H}\right)$ is normal, and thus $\theta \otimes 1: A \rightarrow \mathcal{L}\left(E_{1} \otimes_{\sigma} E_{2}\right)$ is normal.

Lemma 3.6. Let $B$ be a unital von Neumann algebra, let $I$ be a set and for every $\iota \in I$ let $A_{\iota}$ be a unital von Neumann algebra containing a copy of $B$ as a unital von Neumann subalgebra and having a normal conditional expectation $\phi_{\iota}: A_{\iota} \rightarrow B$ whose GNS representation is faithful. Let

$$
(A, \phi)=\underset{\iota \in I}{\bar{*}}\left(A_{\iota}, \phi_{\iota}\right)
$$

be the reduced amalgamated free product of von Neumann algebras. Then for every $\iota_{0} \in I$, there is a normal conditional expectation $\Phi_{\iota_{0}}: A \rightarrow A_{\iota_{0}}$ such 
that $\Phi_{\iota_{0}} \uparrow_{A_{\iota}}=\phi_{\iota}$ for every $\iota \in I \backslash\left\{\iota_{0}\right\}$ and $\Phi_{\iota_{0}}\left(a_{1} a_{2} \cdots a_{n}\right)=0$ whenever $n \geq 2$ and $a_{j} \in A_{\iota_{j}} \cap \operatorname{ker} \phi_{\iota_{j}}$ with $\iota_{1} \neq \iota_{2}, \ldots, \iota_{n-1} \neq \iota_{n}$.

Proof. Let $\psi$ be a normal faithful state on $B$ and let $\tau=\pi_{\psi}: B \rightarrow \mathcal{L}(\mathcal{H})$ be the associated GNS representation. The construction of $A$ can be realized on the Hilbert space $E \otimes_{\tau} \mathcal{H}$. The projection $Q_{\iota_{0}}: E \rightarrow E_{\iota_{0}}$ from the proof of Lemma 1.1 gives rise to the projection $Q_{\iota_{0}} \otimes 1_{\mathcal{H}}: E \otimes_{\tau} \mathcal{H} \rightarrow E_{\iota_{0}} \otimes_{\tau} \mathcal{H}$, compression with which provides a normal positive linear map $\Theta_{\iota_{0}}: A \rightarrow$ $\mathcal{L}\left(E_{\iota_{0}} \otimes_{\tau} \mathcal{H}\right)$. Let $\lambda_{\iota_{0}}: A_{\iota_{0}} \rightarrow \mathcal{L}\left(E_{\iota_{0}} \otimes_{\tau} \mathcal{H}\right)$ be the GNS representation $A_{\iota_{0}} \hookrightarrow \mathcal{L}\left(E_{\iota_{0}}\right)$ followed by the inclusion $\mathcal{L}\left(E_{\iota_{0}}\right) \ni x \mapsto x \otimes 1_{\mathcal{H}} \in \mathcal{L}\left(E_{\iota_{0}} \otimes_{\tau} \mathcal{H}\right)$. Then $\Theta_{\iota_{0}}$ maps a weakly dense *-subalgebra of $A$ into the image of $\lambda_{\iota_{0}}$, hence maps all of $A$ there. Let $\Phi_{\iota_{0}}=\lambda_{\iota_{0}}^{-1} \circ \Theta_{\iota_{0}}$. The desired properties of $\Phi_{\iota_{0}}$ are easily verified.

Here is an embedding result, analogous to Theorem 1.3, for amalgamated free products of von Neumann algebras.

Theorem 3.7. Let $B \subseteq \widetilde{B}$ be a (not necessarily unital) inclusion of von Neumann algebras. Let $I$ be a set and for each $\iota \in I$ suppose

$$
\begin{array}{ll}
1_{\widetilde{A}_{\iota}} \in \widetilde{B} \subseteq & \widetilde{A}_{\iota} \\
\cup & \cup \\
1_{A_{\iota}} \in B & \subseteq A_{\iota}
\end{array}
$$

are inclusions of von Neumann algebras. Suppose that $\tilde{\phi}_{\iota}: \widetilde{A}_{\iota} \rightarrow \widetilde{B}$ is a normal conditional expectation such that $\tilde{\phi}_{\iota}\left(A_{\iota}\right) \subseteq B$ and assume that $\tilde{\phi}_{\iota}$ and the restriction $\tilde{\phi}_{\iota} \uparrow_{A_{\iota}}$ have faithful GNS representations, for all $\iota \in I$. Let

$$
\begin{aligned}
(\tilde{A}, \tilde{\phi}) & =\underset{\iota \in I}{*}\left(\widetilde{A}_{\iota}, \tilde{\phi}_{\iota}\right) \\
(A, \phi) & =\underset{\iota \in I}{*}\left(A_{\iota}, \tilde{\phi}_{\iota} \uparrow_{A_{\iota}}\right)
\end{aligned}
$$

be the amalgamated free products of von Neumann algebras. Then there is a unique normal $*$-homomorphism $\kappa: A \rightarrow \widetilde{A}$ such that for every $\iota \in I$ the diagram

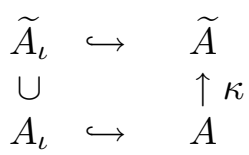

commutes, where the horizontal arrows are the inclusions arising from the free product construction. Moreover, $\kappa$ is necessarily injective.

Proof. This is very much like the proof of Theorem 1.3, to which we refer in detail. Assume without loss of generality that $B$ is a unital subalgebra of $\widetilde{B}$. We now insist that $\tau$ be a normal faithful representation of $\widetilde{B}$; we must show that the algebra homomorphism $\widetilde{\lambda} \circ \widetilde{\sigma}: \mathfrak{A} \rightarrow \mathcal{L}\left(\widetilde{E} \otimes_{\tau} \mathcal{W}\right)$ extends to a 
normal representation of the von Neumann algebra $A$. But $\widetilde{E} \otimes_{\tau} \mathcal{W}$ is the direct sum of $E \otimes_{\tau \uparrow_{B}} \mathcal{W}$ and the various $\widetilde{\mathcal{W}}\left(\iota_{1}, \ldots, \iota_{n}\right)$. The homomorphism $\tilde{\lambda} \circ \tilde{\sigma}$ restricted to $E \otimes_{\tau \uparrow_{B}} \mathcal{W}$ extends to the defining representation of $A$. Let $n \geq 1$ and let $\iota_{1}, \ldots, \iota_{n} \in I$ be such that $\iota_{j} \neq \iota_{j+1}$; we have the normal *-representation, $\mu$, of $A_{\iota_{1}}$ on the Hilbert space $K_{\iota_{1}} \otimes_{\widetilde{B}} E_{\iota_{2}} \otimes_{\widetilde{B}} \cdots \otimes_{\widetilde{B}} E_{\iota_{n}} \otimes_{\tau}$ $\mathcal{W}$, obtained from the normal representation of $A_{\iota_{1}}$ in $\mathcal{L}\left(\widetilde{E}_{\iota_{1}}\right)$; let $\mu l^{A}$ be the representation of the von Neumann algebra $A$ on $\widetilde{\mathcal{W}}\left(\iota_{1}, \ldots, \iota_{n}\right)$ induced from $\mu$ with respect to the normal conditional expectation $\Phi_{\iota_{1}}: A \rightarrow A_{\iota_{1}}$ found in Lemma 3.6; then $\widetilde{\lambda} \circ \widetilde{\sigma}$ restricted to $\widetilde{\mathcal{W}}\left(\iota_{1}, \ldots, \iota_{n}\right)$ extends to the *-homomorphism $\mu l^{A}: A \rightarrow \mathcal{L}\left(\widetilde{\mathcal{W}}\left(\iota_{1}, \ldots, \iota_{n}\right)\right)$, which by Lemma 3.4 is normal.

Here is the construction, analogous to Theorem 2.2, of free products of certain completely positive maps in the von Neumann algebra setting.

Theorem 3.8. Let $B$ be a von Neumann algebra, let $I$ be a set and for every $\iota \in I$ let $A_{\iota}$ and $D_{\iota}$ be von Neumann algebras containing copies of $B$ as unital von Neumann subalgebras and having normal conditional expectations $\phi_{\iota}: A_{\iota} \rightarrow B$, respectively $\psi_{\iota}: D_{\iota} \rightarrow B$, whose GNS representations are faithful. Suppose that for each $\iota \in I$ there is a normal unital completely positive map $\theta_{\iota}: A_{\iota} \rightarrow D_{\iota}$ that is also a $B-B$ bimodule map and satisfies $\psi_{\iota} \circ \theta_{\iota}=\phi_{\iota}$. Let

$$
\begin{aligned}
& (A, \phi)=\underset{\iota \in I}{\bar{*}}\left(A_{\iota}, \phi_{\iota}\right) \\
& (D, \phi)=\underset{\iota \in I}{\underset{*}{*}}\left(D_{\iota}, \psi_{\iota}\right)
\end{aligned}
$$

be the reduced amalgamated free products of von Neumann algebras. Then there is a normal unital completely positive map $\theta: A \rightarrow D$ satisfying

$$
\forall \iota \in I \quad \theta \uparrow_{A_{\iota}}=\theta_{\iota}
$$

and

$$
\theta\left(a_{1} a_{2} \cdots a_{n}\right)=\theta\left(a_{1}\right) \theta\left(a_{2}\right) \cdots \theta\left(a_{n}\right)
$$

whenever $a_{j} \in A_{\iota_{j}} \cap \operatorname{ker} \phi_{\iota_{j}}$ and $\iota_{1} \neq \iota_{2}, \iota_{2} \neq \iota_{3}, \ldots, \iota_{n-1} \neq \iota_{n}$.

Proof. Let

$$
\begin{aligned}
& (\mathcal{A}, \varphi)=\underset{\iota \in I}{*}\left(A_{\iota}, \phi_{\iota}\right) \\
& (\mathcal{D}, \rho)=\underset{\iota \in I}{*}\left(D_{\iota}, \psi_{\iota}\right)
\end{aligned}
$$

be the $\mathrm{C}^{*}$-algebra reduced amalgamated free products. Thus $A$ and $D$ are the closures in strong-operator topology of $\mathcal{A}$ and respectively $\mathcal{D}$ in the appropriate representations. We need only show that the unital completely positive map $\theta: \mathcal{A} \rightarrow \mathcal{D}$ found in Theorem 2.2 extends to a normal completely positive map $\bar{\theta}: A \rightarrow D$. 
Consider, from the proof of Theorem 2.2, the Hilbert $B$-modules $(E, \xi)=$ $\underset{\iota \in I}{*}\left(E_{\iota}, \xi_{\iota}\right)$, and $(F, \eta)=\underset{\iota \in I}{*}\left(F_{\iota}, \eta_{\iota}\right)$, the $*$-homomorphism $\sigma: \mathcal{A} \rightarrow \mathcal{L}(F)$ and the bounded operator $v \in \mathcal{L}(E, F)$; denote by $i_{\mathcal{A}}$ the GNS representation of $\mathcal{A}$ on $L^{2}(\mathcal{A}, \varphi)$. Recall that $\sigma$ is a free product of embeddings $\sigma_{\iota}: A_{\iota} \rightarrow$ $\mathcal{L}\left(F_{\iota}\right)$. From the proof of Theorem 1.3, and letting $\tau=\pi_{\mu}: B \rightarrow \mathcal{L}(\mathcal{V})$ be the GNS representation of a normal faithful state $\mu$ of $B$, we see that the representation $\sigma \otimes 1$ of $\mathcal{A}$ on $F \otimes_{\tau} \mathcal{V}$ given by $a \mapsto \sigma(a) \otimes 1$ splits as a direct sum, $\sigma \otimes 1=\bigoplus_{\lambda \in \Lambda}(\sigma \otimes 1)\left\lceil_{\mathcal{W}_{\lambda}}\right.$, where each summand $(\sigma \otimes 1) \Upsilon_{\mathcal{W}_{\lambda}}$ is either a copy of $i_{\mathcal{A}} \otimes 1: \mathcal{A} \rightarrow \mathcal{L}\left(L^{2}(\mathcal{A}, \varphi) \otimes_{\tau} \mathcal{V}\right)$ or is the induced representation $\nu l^{\mathcal{A}}$ of a representation $\nu$ of some $A_{\iota}$ on a Hilbert space, where $\nu$ is the restriction to an invariant subspace of the representation $\sigma_{\iota} \otimes 1: A_{\iota} \rightarrow \mathcal{L}\left(F_{\iota} \otimes_{\tau} \mathcal{V}\right)$. The representation $i_{\mathcal{A}} \otimes 1$ extends to a normal $*$-representation of $A$ as seen in Construction 3.1. Using Lemma 3.3 we see that $\sigma_{\iota} \otimes 1$ is normal; hence $\nu$ is normal and by Lemma 3.4, $\nu l^{\mathcal{A}}$ extends to a normal $*$-representation of $A$. Hence $\sigma \otimes 1$ extends to a normal *-representation of $A$, which we will denote by $\bar{\sigma}: A \rightarrow \mathcal{L}\left(F \otimes_{\tau} \mathcal{V}\right)$.

The isometry $v \in \mathcal{L}(E, F)$ gives rise to an isometry $v \otimes 1: E \otimes_{\tau} \mathcal{V} \rightarrow F \otimes_{\tau} \mathcal{V}$. Letting $i_{D}: \mathcal{D} \rightarrow \mathcal{L}(E)$ be the defining representation, by Remark 3.2 the weak closure of the image of $i_{D} \otimes 1: \mathcal{D} \rightarrow \mathcal{L}\left(E \otimes_{\tau} \mathcal{V}\right)$ is the von Neumann algebra $D$. Consider the normal unital completely positive map $\bar{\theta}: A \rightarrow$ $\mathcal{L}\left(E \otimes_{\tau} \mathcal{V}\right)$ given by $\bar{\theta}(x)=(v \otimes 1)^{*} \bar{\sigma}(x)(v \otimes 1)$. If $a \in \mathcal{A}$ then $\bar{\theta}(a)=$ $i_{D}(\theta(a)) \otimes 1$. So $\bar{\theta}$ extends the map $\theta: \mathcal{A} \rightarrow \mathcal{D}$; hence $\bar{\theta}(A) \subseteq D$.

\section{References}

[1] D. Avitzour, Free products of $C^{*}$-algebras, Trans. Amer. Math. Soc., 271 (1982), 423-465, MR 83h:46070, Zbl 513.46037.

[2] F. Boca, Free products of completely positive maps and spectral sets, J. Funct. Anal., 97 (1991), 251-263, MR 92f:46064, Zbl 741.46024.

[3] Completely positive maps on amalgamated free product $C^{*}$-algebras, Math. Scand., 72 (1992), 212-222, MR 94k:46110.

[4] M. Bożejko, M. Leinert and R. Speicher, Convolution and limit theorems for conditionally free random variables, Pacific J. Math., 175 (1996), 357-388, MR 98j:46069, Zbl 874.60010.

[5] M. Bożejko and R. Speicher, $\psi$-independent and symmetrized white noises in 'Quantum Probability \& Related Topics, QP-PQ VI', 219-236, World Sci. Publishing, 1991, CMP 1149828.

[6] W.-M. Ching, Free products of von Neumann algebras, Trans. Amer. Math. Soc., 178 (1973), 147-163, MR 48 \#4749.

[7] M. Choda, Reduced free products of completely positive maps and entropy for free product of automorphisms, Publ. Res. Inst. Math. Sci., 32 (1996), 371-382, MR 97d:46077, Zbl 857.46040.

[8] M. Choda and K.J. Dykema, Purely infinite simple $C^{*}$-algebras arising from free product constructions, III, Proc. Amer. Math. Soc., 128(11) (2000), 3269-3273, MR 2001b:46095. 
[9] K.J. Dykema, Faithfulness of free product states, J. Funct. Anal., 154 (1998), 323-329, MR 99e:46066, Zbl 927.46034.

[10] Simplicity and the stable rank of some free product $C^{*}$-algebras, Trans. Amer. Math. Soc., 351 (1999), 1-40, MR 99g:46078.

[11] _ Purely infinite simple $C^{*}$-algebras arising from free product constructions, II, Math. Scand., to appear.

[12] _ Exactness of reduced amalgamated free product $C^{*}$-algebras, preprint, 1999.

[13] _ - Topological entropy of some automorphisms of reduced amalgamated free product $C^{*}$-algebras, Ergodic Theory Dynam. Systems, to appear.

[14] K.J. Dykema, U. Haagerup and M. Rørdam, The stable rank of some free product $C^{*}$-algebras, Duke Math. J., 90 (1997), 95-121, MR 99g:46077a; Correction, idem., 94 (1998), 213, Zbl 943.46034.

[15] K.J. Dykema and M. Rørdam, Purely infinite simple $C^{*}$-algebras arising from free product constructions, Canad. J. Math., 50 (1998), 323-341, MR 99d:46074, Zbl 919.46040.

[16] MR 99d:46075, Zbl 907.46045; Erratum, idem., 10(4) (2000), 975, CMP 1791146.

[17] G.G. Kasparov, Hilbert $C^{*}$-modules: Theorems of Stinespring and Voiculescu, J. Operator Theory, 4 (1980), 133-150, MR 82b:46074, Zbl 456.46059.

[18] E.C. Lance, Hilbert $C^{*}$-modules, a Toolkit for Operator Algebraists, London Math. Soc. Lecture Note Series, 210, Cambridge University Press, 1995, MR 96k:46100, Zbl 822.46080.

[19] M.A. Rieffel, Induced representations of $C^{*}$-algebras, Adv. Math., 13 (1974), 176-257, MR 50 \#5489, Zbl 284.46040.

[20] Morita equivalence for $C^{*}$-algebras and $W^{*}$-algebras, J. Pure Appl. Algebra, 5 (1974), 51-96, MR 51 \#3912, Zbl 295.46099.

[21] D. Voiculescu, Symmetries of some reduced free product $C^{*}$-algebras, Operator Algebras and Their Connections with Topology and Ergodic Theory, Lecture Notes in Mathematics, 1132, Springer-Verlag, (1985), 556-588, MR 87d:46075, Zbl 618.46048.

Received June 10, 1999 and revised September 29, 2000. The second author was partially supported as an invited researcher funded by CNRS of France and by NSF Grant No. DMS 0070558.

Institut de Mathématiques De Luminy

163, AVEnue De Luminy, CASE 907

F-13288 MARSEILle

FRANCE

E-mail address: blanch@iml.univ-mrs.fr

Dept. of Mathematics Texas A\&M University

College Station TX 77843-3368

E-mail address: Ken.Dykema@math.tamu.edu

The Mathematical Sciences Research Institute

1000 Centennial Drive, \#5070

Berkeley CA 94720-5070 